\title{
A Study on the Validity of Commodity Evaluation of E-commerce Platform Based on Dynamic Transaction Events
}

\author{
Bingwen Yan ${ }^{1,2, a}$, Chunqiong $\mathrm{Wu}^{1,2}$ and Rongrui $\mathrm{Yu}^{1,2}$ \\ ${ }^{1}$ Yango University, Business and Marketing Department, Fuzhou, Fujian, China \\ ${ }^{2}$ Engineering Research Center of Business Intellgent in Big Data for Fujian Province
}

\begin{abstract}
Commodity evaluation on e-commerce platforms affects the sale of goods and the behavior of buyers. Although the observation point of static event theory is stable, it emphasizes one-sided data too much. It ignores the interconnections in the development of events and makes it difficult to effectively analyze the validity of commodity evaluation behaviors. Under the concept of dynamic transaction events based on dynamic development, the validity of commodity evaluation is calculated from time, space and intensity factors. This can consider the influence of individual evaluator and overall evaluator to evaluate the validity of commodity evaluation systematically and objectively.
\end{abstract}

Keywords: Dynamic transaction events; E-commerce platform; Commodity evaluation.

\section{Introduction}

The combination of information technology and Internet technology has formed many mature e-commerce platforms, which can help strangers to conduct transactions across regions. The third-party e-commerce platform plays an endorsement role in the transactions between strangers, and adopts user evaluation mechanism of merchants and commodities to restrict bad transaction behavior. However, the validity of the evaluation scores is not well achieved. On the one hand, there is a malicious water army deliberately lowering or raising the commodity evaluation; On the other hand, the network customers' own feelings, habits, tendencies and other factors are sparsely distributed. These are not conducive to a credible commodity evaluation. In order to correctly evaluate the quality of commodities and effectively explore the intrinsic value of commodity evaluation, several important factors affecting commodity evaluation can be identified from the perspective of dynamic transaction events.

\section{The concept of dynamic transaction events}

When many scholars study the occurrence of an event, they usually regard the event as a set of stagnant and completed data sets. It is true that any event exists independently under the definitions of time axis or specific conditions, and is relatively static. It has the

a Corresponding author:bingwen_yan@163.com advantages of stability, easy quantification and easy interpretation. But "static events" separated from the time axis can only be represented as a miniature of the data set on the time axis. It only shows the one-sided stability of the entity, so we can not deduce the understanding of the whole event by partial generalization. At the same time, the data set of static events lacks the influence of interaction with other entity factors continuously and interactively.

This is especially true when the transaction behavior of e-commerce is viewed from the perspective of event theory. This is because e-commerce transaction behavior is interrelated with people, platforms, third-party logistics, Internet users, social trends, etc., which are intertwined and trigger each other and affect the whole body. Thus, some attributes of transaction behavior should not be isolated for analysis.

Scholar Liu Dong ${ }^{[1]}$ creates the theory of event systems. He thinks that most of the existing researches are static researches on feature-oriented while dynamic researches on event-oriented are lacking. This paper mainly adopts Liu Dong's point of view and regards the commodity evaluation behavior of e-commerce platforms as a dynamic event. The vitality of the commodity evaluation are predicted by evaluating the interaction of the commodity evaluation entity's characteristics with the event, the entity's stable feature variable interacting with the novelty, subversive event, key event, or event's overall intensity experienced. 


\section{Single commodity evaluation $L_{i j}$}

Commodity evaluation has strong subjectivity, which is closely related to the buyer's own needs, expectations and sense of self-realization. This can be said to vary from person to person and is a fuzzy indicator. Most of the current e-commerce platforms adopt the 5-point system. The performance value of commodity evaluation is obtained after a comprehensive score is made on whether the commodity meets the buyer's expectation, logistics satisfaction and service satisfaction. In addition, the evaluation text is also one of the important parameters of commodity evaluation, which can better eliminate the evaluation distortion caused by contradictory evaluation ${ }^{[2]}$. Some buyers are more casual in the evaluation of goods, so that the evaluation does not have reference value. For example, a large amount of repetitive evaluation information indicates that the buyer's evaluation is of low usefulness $^{[3]}$. Most buyers will consider the evaluation information of the product as one of the indicators of whether to buy before purchasing. However, some buyers do not complete the evaluation of the commodity in time or seriously after the purchase ${ }^{[4]}$. This part of the evaluation data also has an impact on the vitality of the final evaluation of the commodity. However, in any case, the buyer's expectation of commodity attribute ${ }^{[5]}$ is the first factor that influences commodity evaluation. Open commodity evaluation will ultimately affect the sale of commodities $^{[6]}$.

An e-commerce transaction is not a static event whose parameters are no longer changing. On the contrary, each step is a new and dynamic transaction event, from the initiation and implementation of the initial transaction, to the generation of transaction results, and even to the subsequent transaction or other transaction behaviors further affected by evaluation factors. Dynamic transaction events change with time and the process of event development, and form new factors that influence the trend of events themselves.

In the concept of dynamic transaction event, a commodity evaluation behavior is a dynamically changing event. The occurrence of events has factors of time $H_{i j}$ and space $P_{i j}$. The development and change of events are determined by the intensity ${ }_{i j}$ of events.

That is, corresponding to the factor that forms the evaluation $L_{i j}$ of the buyer $\mathrm{i}$ on the item $\mathrm{j}$, it is expressed as:

$$
L_{i j}=\left\{\begin{array}{cc}
0.5 & n=0 \\
\sum_{l=1}^{n} S_{i j} \cdot H_{i j} \cdot P_{i j} & n \geq 1
\end{array}\right.
$$

(Formula 1)

Time factor $H_{i j}$

For dynamic transaction events, the time factors of commodity evaluation are as follows. This includes whether the commodity evaluation is timely, whether there is conflicting information between the commodity evaluation and the follow-up evaluation, and whether the commodity evaluation has an impact on the commodity sales due to the change of time.

The main factors influencing commodity evaluation in terms of time factors include the following: The length of time it takes for the buyer to initiate a purchase until the purchase is completed $H P_{i j}$, The length of time of commodity evaluation after confirmation of receipt $\mathrm{H} I_{i j}$, The length of the interval between the second evaluation and the first evaluation $\mathrm{H} F_{i j}$, factors whether the follow-up evaluation is contradictory with the first evaluation $\mathrm{HR}_{i j}$, the impact of commodity evaluation on sales $\mathrm{HM}_{i j}$.

That is, when the buyer $\mathrm{i}$ makes the $\mathrm{fth}$ commodity evaluation of the commodity $\mathrm{j}$, the time factor is recorded ${ }_{\text {as }} H_{i j}$

$$
\begin{aligned}
& H_{i j}=H P_{i j}^{f} \cdot H I_{i j}^{f} \cdot H F_{i j}^{f} \cdot H R_{i j}^{f} \cdot H M_{i j}^{f} \text { (Formula 2) } \\
& \text { Space factor } P_{i j}
\end{aligned}
$$

For dynamic transaction events, the space factors of commodity evaluation are as follows: The degree of dissemination of commodity evaluation, the initial influencing factors of commodity evaluation, the physical distance between a commodity evaluator and the commodity and the physical space distance between the product evaluator and the evaluation browser, etc.

Therefore, the main factors affecting commodity evaluation in terms of space factors include: The number of browses and comments on commodity evaluation $P S_{i j}$, the initial influencing factors of commodity evaluation $P F_{i j}$, spatial physical distance between commodity evaluator and commodity delivery place $P C_{i j}$, spatial physical distance between commodity evaluator and browser $P W_{i j}$

That is, when the buyer $\mathrm{i}$ makes the $\mathrm{fth}$ commodity evaluation of the item $\mathrm{j}$, the space factor is recorded $P_{i j}$ :

$$
P_{i j}=P S_{i j}^{f} \cdot P C_{i j}^{f} \cdot P W_{i j}^{f}
$$

(Formula 3)

Intensity factor $S_{i j}$

For dynamic transaction events, the intensity factors of commodity evaluation are as follows: Whether the commodity evaluation has novelty, whether it has the value of typical parameters, and whether it affects the commodity evaluation score, and whether the purchase amount has an impact on commodity evaluation, etc.

Therefore, in terms of intensity factors, the main factors affecting commodity evaluation include: Whether the buyer evaluates the commodity for the first time $S F_{i j}$, whether the indicator of single commodity evaluation has 
obvious tendency $S T_{i j}$, whether a single commodity evaluation forms a inter-reference with the evaluation of other buyers $S R_{i j}$, amount of single purchase $S M_{i j}$ and whether there is any follow-up evaluation behavior $S A_{i j}$.

That is, when the buyer $\mathrm{i}$ makes the $\mathrm{fth}$ commodity evaluation of the item $\mathrm{j}$, the intensity factor $S_{i j}$ is recorded as:

$$
S_{i j}=S F_{i j}^{f} \cdot S T_{i j}^{f} \cdot S R_{i j}^{f} \cdot S M_{i j}^{f} \cdot S A_{i j}^{f} \quad \text { (Formula 4) }
$$

Among the factors that constitute buyer i's evaluation $L_{i j}$ of commodity $\mathrm{j}$, most of them are uncertain and subjective. For example, it includes the length of time $\mathrm{H} I_{i j}$ between commodity evaluation after confirmation of receipt, the number $P S_{i j}$ of browses and comments on commodity evaluation and whether there is any follow-up evaluation behavior $S A_{i j}$. These factors exist objectively, but the samples are sparsely distributed, which is difficult to explain by statistical methods. Therefore, it is necessary to further understand the correlation between commodity evaluator $\mathrm{i}$ and other buyers $\mathrm{j}$ who also buy this commodity.

\section{Overall commodity evaluation ${ }^{L_{j}}$}

When the commodity exists on the e-commerce platform, it is a state of open evaluation. All buyers who have purchased the commodity are allowed to evaluate it. It can be seen that for the evaluation of one commodity $\mathrm{j}$, it is necessary to consider not only the buyer i's single commodity evaluation, but also the evaluation of other buyers who have purchased the product $\mathrm{j}$. In fact, the correlation between commodity evaluators and commodities also affects the vitality of commodity evaluation. In the overall commodity evaluation, the evaluators are divided into the buyer $\mathrm{r}$ with higher commodity correlation (quantity denoted as $\mathrm{N}$ ) and the buyer $\mathrm{k}$ with lower commodity correlation (quantity denoted as $\mathrm{M}$ ). The correlation is obtained by a combination of the number of purchases of the commodity $\rho S_{r}$, the number of purchases of similar commodities $\rho C S_{r}$, and the types of purchases of similar commodities $\rho T S_{r}$. The total number of evaluators is $(\mathrm{r}+\mathrm{k})$, and the evaluation of commodity $\mathrm{j}$ is $L_{r j}$ and $L_{k j}$, respectively.

That is, corresponding to the evaluation ${ }^{r j}$ of commodity $\mathrm{j}$ by buyer $\mathrm{r}$ with a high degree of correlation, when the weighting factor is $\rho_{r}$, there is:

$$
L_{r j}=\frac{\sum_{r=1}^{N}\left(\rho_{r} \cdot l_{r j}\right)}{\sum_{r=1}^{N} \rho_{r}}
$$

Corresponding to the evaluation ${ }^{L_{k j}}$ of commodity $\mathrm{j}$ by buyer $\mathrm{k}$ with a low degree of correlation, when the weighting factor is $\rho_{k}$, there is:

$$
L_{k j}=\frac{\sum_{k=1}^{M}\left(\rho_{k} \cdot L_{k j}\right)}{\sum_{k=1}^{N} \rho_{k}}
$$

$\rho_{r \text { and }} \rho_{k}$ represent the degree of correlation between the buyer and the commodity. After $\mathrm{f}$ purchases, high correlation $\rho_{r}$ is expressed as:

$$
\rho_{r}=\rho S_{r}^{f} \cdot \rho C S_{r}^{f} \cdot \rho T S_{r}^{f}
$$

(Formula 7)

After $\mathrm{f}$ purchases, low correlation $\rho_{k}$ is expressed as:

$$
\rho_{k}=\rho S_{k}^{f} \cdot \rho C S_{k}^{f} \cdot \rho T S_{k}^{f}
$$

To sum up, it can be concluded that the overall commodity evaluation $L_{j}$ can be expressed as:

$$
L_{j}=\frac{\sum_{i=1}^{N} L_{r j}}{N}+\frac{\sum_{i=1}^{M} L_{k j}}{M}
$$

\section{Validity of commodity evaluation}

The vitality of commodity evaluation $T_{i j}$ is formed ( $T_{i j} \in[0,1]$ ) after calculating the single commodity evaluation ${ }^{L}{ }$ of buyer $\mathrm{i}$ on commodity $\mathrm{j}$ and the overall commodity evaluation $L_{j}$ obtained through the open evaluation platform. It can help other potential commodity buyers to have a better sense of experience, and decide whether the purchase behavior is generated and carried out.

The confidence factor $\beta$ represents the degree of trust of a specific buyer $\mathrm{i}$ in the overall commodity evaluation ${ }^{L}{ }_{j}$ of commodity $\mathrm{j}$.

That is, the confidence factor $\beta$ is:

$$
\beta=\frac{\sum M_{f}}{\sum_{\text {total }=1}^{n} M_{\text {total }}}
$$

(Formula 10)

$M_{f}$ is the total amount of money generated by commodity $\mathrm{j}$ in the $\mathrm{f}$ th transaction. Here, if we do not use 
the amount of money, but the number of times to measure, not only the amount of data will be bigger, but also can not reflect the importance of the amount in the commodity attributes. $M_{\text {total }}$ is the total amount of historical transactions of commodity $\mathrm{j}$.

To sum up, the vitality of commodity evaluation $T_{i j}$ can be expressed as follows:

$$
T_{i j}=e^{-\frac{n}{N}} \cdot L_{i j}+\left(1-e^{-\frac{n}{N}}\right) \cdot \beta \cdot L_{j}
$$

(Formula 11)

\section{Conclusion and suggestion}

In fact, data from e-commerce platforms is not standard quantitative data and structured data. Therefore, before analyzing the vitality of commodity evaluation, the data should be standardized and cleared according to the actual situation of the commodity, so that the scale of data measurement is unified and suitable for calculation.

Cluster analysis is a common and typical data mining algorithm. According to the clustering classifier, the input training data of commodity evaluation are studied, and the central point of the clustering subgroup is obtained. Furthermore, the distance between the test data and each central point is calculated based on the central point data of the cluster subgroup. Then, the difference between the commodity evaluation of a single data set and the average commodity evaluation is obtained. When the commodity evaluation score of a single data set is greater than the average commodity evaluation score, the commodity evaluation is better, and vice versa. The size of clustering subset represents the value of commodity evaluation.

The evaluation of a commodity is realized by integrating the common opinions of individual buyers and group buyers. In economic theory, the expensive transaction amount is more likely to make the buyer have psychological crisis after purchase, which has an impact on the vitality of commodity evaluation. Therefore, not only does the money play a role in the vitality of commodity evaluation, but it is also a requirement for e-commerce merchants. E-commerce merchants should do a good job in after-sales service and commodity tracking service to ensure that buyers can produce correct and objective commodity evaluation.
In general, the evaluation value of a commodity is a vague, constantly changing outcome, or a process that further affects. The analysis of the vitality of commodity evaluation constructed by the concept of dynamic transaction events fully considers the dynamic factors in the process of commodity evaluation. From the time, space and intensity factors, it analyzes the various factors affecting commodity evaluation and constructs a calculation model for the vitality of commodity evaluation. Scientific and rational commodity evaluation management can avoid the subjective randomness of confidence indicators and promote the regulation of e-commerce transactions.

\section{Acknowledgement}

The project name of the fund: College-level project of Yango University "Study on E-commerce Trust Evaluation Model Based on Dynamic Transaction Event Horizon in Big Data Environment".

\section{References}

1. Liu Dong. Event System Theory: An Event-oriented Approach to the Organizational Sciences [J]. Academy of Management Review, 2015, 40(04): 515-537.

2. Wang Qian, $\mathrm{Fu}$ Kui. Calculation Method of Comprehensive Score of Commodity Evaluation Based on LSTM-AE Neural Network [J]. Journal of Beijing University of Posts and Telecommunications (Social Science Edition), 2018, (04): 19-27.

3. Li Jianchao. Research on the Usefulness of Online Product Evaluation [D]. Jinan University, 2018.

4. Liu Mengyuan. Research on the User Evaluation System of Taobao [D]. Hebei University, 2018.

5. You Tianhui, Zhang Jin and Fan Zhiping. Method for Selecting Desirable Product(s) Based on Online Rating Information and Consumer's Aspirations [J]. Chinese Journal of Management Science, 2017, (11): 94-102.

6. Sun Jiasheng. Integrating Multiple Opinions_-Strategic Display of Product Ratings [J]. China Soft Science, 2017, (08): 184-192. 